\title{
Segmentation and Size Measurement of Polyps in CT Colonography
}

\author{
J.J. Dijkers ${ }^{1,2}$, C. van Wijk ${ }^{1}$, F.M. Vos ${ }^{1,3}$, J. Florie ${ }^{3}$, Y.C. Nio ${ }^{3}$, \\ H.W. Venema ${ }^{3,4}$, R. Truyen ${ }^{2}$, and L.J. van Vliet $^{1}$ \\ 1 Quantitative Imaging Group, Department of Imaging Science and Technology, \\ Faculty of Applied Sciences, Delft University of Technology, Delft, The Netherlands \\ L.J.vanVliet@tnw.tudelft.nl http://www.qi.tnw.tudelft.nl/ \\ 2 Philips Medical Systems, Best, The Netherlands \\ 3 Department of Radiology, Academic Medical Center, Amsterdam, The Netherlands \\ 4 Department of Medical Physics, Academic Medical Center, \\ Amsterdam, The Netherlands
}

\begin{abstract}
Virtual colonoscopy is a relatively new method for the detection of colonic polyps. Their size, which is measured from reformatted CT images, mainly determines diagnosis. We present an automatic method for measuring the polyp size. The method is based on a robust segmentation method that grows a surface patch over the entire polyp surface starting from a seed. Projection of the patch points along the polyp axis yields a 2D point set to which we fit an ellipse. The long axis of the ellipse denotes the size of the polyp. We evaluate our method by comparing the automated size measurement with those of two radiologists using scans of a colon phantom. We give data for inter-observer and intra-observer variability of radiologists and our method as well as the accuracy and precision.
\end{abstract}

\section{Introduction}

Colorectal cancer is one of the most commonly diagnosed types of cancer. Specifically, the American Cancer Society predicts 145,000 new cases and 56,000 deaths from colorectal cancer for 2005 [1. Polyps are a well-known precursor to such carcinoma. Not surprisingly, it has been shown that early removal of polyps ensures a decrease in incidence [7].

In recent years, CT colonography has been proposed as a noninvasive alternative to traditional polyp detection by colonoscopy $[9,5$. In CT colonography, the colon structure is often visualized from an endoluminal perspective by means of surface or volume rendering. Recently, methods have been proposed to support the inspection by a computer aided detection (CAD) system indicating suspect locations [8, 4. The size of a detected polyp is an important aspect for diagnosis and decision making. It is generally accepted that polyps with diameter i $5 \mathrm{~mm}$ require no direct further action, whereas larger polyps should be removed via colonoscopy. Typically, the size of polyps is measured in colonoscopy by comparison with an open biopsy forceps. In CT colonography, it is usually measured in reformatted images, in which the largest polyp diameter is selected for size 
measurement. However, polyp sizes thus measured by human experts can show significant inter- and intra-observer variability.

Clearly, an automated method is needed to enable more accurate measurement of polyp size. As a side effect, such a procedure is also useful in CAD algorithms. Automated polyp detection is usually based on sophisticated pattern recognition techniques that take into account many features measured on tentatively selected candidates (e.g. size, area, average shape index etcetera). Proper segmentation is crucial to perform reliable feature measurement.

The existing methods for colonic polyp segmentation (such as Summers et al. [3, 2, and Yoshida et al. [6]) are especially designed to work directly on the 3D CT data. Such an approach is hindered by not operating on a specifically defined region of interest c.q. the colon surface. Hence, segmentation of polyps which are by definition protrusions of the colon surface is not a trivial task.

In this paper we present a new method for semi-automatic segmentation of polyp-like structures. Additionally, a technique is described to automatically measure polyp sizes using this algorithm. Our method assumes that the colon surface has been identified as a region of interest. Moreover, it is asserted that a candidate location has been identified; in our system by a vertex detection step based on the measured shape index 10. We will compare the size measurement by our algorithm with that of physicians in a set of phantom objects (in which the size is known a priori).

\section{Method}

The description below assumes that the colon wall is described by a triangular mesh. However, the basic ideas of the method are not restricted to a mesh based surface representation of the colon wall; they can as well be implemented to work on a voxel based model. An additional advantage of our method is that the segmentation requires no user input, that is all parameters are drawn from the underlying data.

Outline of segmentation procedure. Ideally, a polyp could be described as a rather spherical, symmetric mound on a background shape (see e.g. Figure 2 a). One could intuitively delineate a polyp by the inflection points on both sides. However, these points may not be easily identifiable due to the curvature of the background shape (think of a fold).

Hence, we model a polyp to have a symmetry axis that goes through the center point $\left(P_{c}\right)$ in which the apical surface normals converge, and the mean position $\left(P_{m}\right)$ calculated from the polyps surface points. The edge of the polyp is defined by the points at which the surface normals tend to deflect from the center point (we will formalize this below).

Initially, a single position or a small patch indicates a point on the polyp candidate [10]. Since the center and mean points may not be robustly determined from such a seed patch, the polyp segmentation procedure is set up as an iterative process. During each cycle of this process neighboring vertices are added if certain 


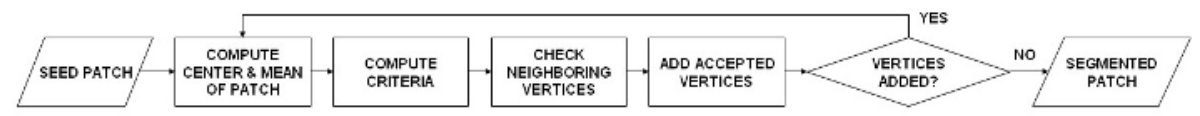

Fig. 1. Schematic overview of segmentation procedure

criteria are met. The process terminates when no more points are added. An overview of the procedure is shown in figure 1

Computing the center and mean points. As depicted in Figure 2a, the surface normals on the polyp apex tend to converge in a center point. This point $\left(P_{c}\right)$ is found by minimizing the sum of the distances $\left(d_{i}\right)$ to all normals $\left(\boldsymbol{n}_{i}\right)$. The surface normals are calculated by Gaussian derivatives the underlying 3D CT data at a scale of $2 \mathrm{~mm}$. This scale was determined experimentally such that no polyps are missed. The distances can be computed according to:

$$
d_{i}=\left\|\boldsymbol{n}_{\boldsymbol{i}} \times\left(P_{i}-P_{c}\right)\right\| /\left\|\boldsymbol{n}_{i}\right\|
$$

where $P_{i}$ is a point on the patch and $\mathrm{x}$ denotes the vector outer product. Additionally, a mean point $\left(P_{m}\right)$ is associated with a patch. The position of the mean is simply computed by averaging the positions of all vertices: $P_{m}=\frac{1}{N} \sum P_{i}$. The mean and the center points define a centerline (dashed in Figure 2b). Henceforth it is called the polyp axis.

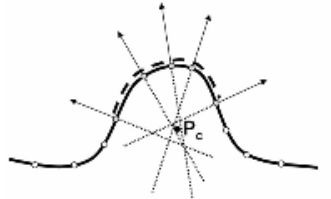

(a)

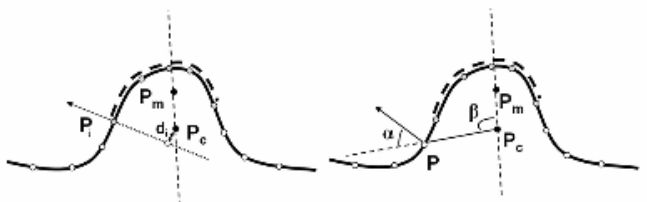

(b)

(c)

Fig. 2. Schematic representation of a patch (dashed curve) on the colon wall. Figure (a) shows how convergent normals define a center point; figure (b) shows how the minimized distance $d_{i}$ is defined for surface point $P_{i}$; figure (c) shows how the angles $\alpha$ and $\beta$ are defined.

Adding points to a seed patch Points are to be added to a seed patch until the local surface normal tends to deviate from $P_{c}$. To formalize the stopping criterion, consider first a sphere on a flat background. Let us define $\alpha$ as the angle between the line from the center point $\left(P_{c}\right)$ to the vertex $\left(P_{i}\right)$ and the normal at the position of the vertex (see Figure 2 ). Clearly, on top of the polyp $\alpha$ is small (exactly zero on a spherical cap, see Figure 3). The angle $\alpha$ increases while moving to periphery of the polyp. Right outside the polyp the angle is given by (compare with Figure [3):

$$
\alpha_{\text {edge }}=\arccos \left[\frac{\left.P_{\text {edge }}-P_{c}\right) \cdot \boldsymbol{n}}{\left\|P_{\text {edge }}-P_{c}\right\| \cdot\|\boldsymbol{n}\|}\right]
$$


in which $P_{\text {edge }}$ is defined as in Figure 3 and $\boldsymbol{n}$ is the normal at point $P_{\text {edge }}$. We assume that the ideal threshold-value lies somewhere between these extreme values (respectively 0 and $\alpha_{e d g e}$ ). The required midway point is closely approximated by the angle calculated via (compare with Figure 3 ):

$$
\alpha_{m i d}=\arccos \left[\left(P_{m}-P_{c}\right) / R\right]
$$

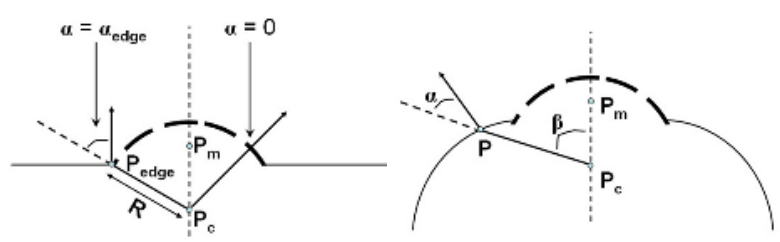

Fig. 3. Schematic representation of a polyp (dashed curve) on a flat background (left) and on a fold (right)

Thus, $\alpha<\alpha_{\text {mid }}$ yields a safe stopping criterion for adding neighboring vertices to a polyp on a flat background. On a fold, however, the angle $\alpha$ remains small (see Fig. 3). Let us define $\beta$ as the angle between the polyp axis and the line between the vertex and the center point (as in Figure 2 2 ):

$$
\beta=\arccos \left[\frac{\left(P-P_{c}\right) \cdot\left(P_{m}-P_{c}\right)}{\left\|P-P_{c}\right\| \cdot\left\|P_{m}-P_{c}\right\|}\right]
$$

At the edge of the polyp $\beta$ is given by $\beta_{e d g e}=\alpha_{e d g e}$. Typically, $\beta$ continues to increase while moving onto the fold. Consequently, $\beta<\beta_{\text {edge }}$ yields a logical stopping criterion for a polyp on a fold. It should be noticed that the two posed criteria are mutually exclusive: the sidepoints of a polyp on a fold do not meet the criterion of $\alpha<\alpha_{\text {mid }}$. On the other hand, points besides a polyp on flat

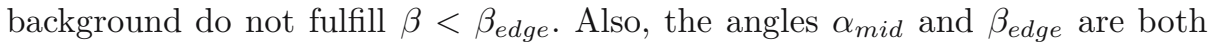
dependent on the shape of a polyp. Flatter polyps tend to have lower values for $\alpha_{m i d}$ and $\beta_{\text {edge }}$ than more protruding polyps. In other words, the threshold values automatically depend on the polyp shape.

All vertices neighboring a seed patch that match the conditions are accepted and added at once to yield a new seed. Consequently, the outcome does not depend on the order in which points are processed. Clearly, if none of the vertices match the criteria, no points are added and the current patch is considered the final, segmented polyp. Otherwise, all steps are iterated.

Automated size measurement. The size measurements for polyps are based upon the segmented patches. The edges of these patches are projected along the polyp axis onto a plane. An ellipse is fitted to these points in $2 \mathrm{D}$ space by computation of the first and second order moments. This is in accordance with the current medical practice in the Academic Medical Center where the polyp size is characterized by its largest diameter. 


\section{$3 \quad$ Experiments and Results}

The performance of the method was assessed by comparing the automated size measurement with those of radiologists using scans of a colon phantom. We have looked into several aspects to test our approach:

- Inter-observer variability of radiologists

- Intra-observer variability of radiologists and our method

- Accuracy and precision of the radiologists and our method

Experimental data. All data was acquired using a Mx8000 multislice CTscanner (Philips Medical Systems, Best, the Netherlands) using the same scanning protocol for all scans (scan parameters: $120 \mathrm{kV}, 100 \mathrm{mAs}, 4$ x $2.5 \mathrm{~mm}$ collimation, pitch 1.25, standard reconstruction filter, and a 180o interpolation algorithm).

The phantom consisted of a lucite cylinder into which fabricated polyps were inserted. At first, the phantom contained 10 hemispherical lucite objects of various sizes, and 4 objects with reduced height $(2 \mathrm{~mm})$ in order to mimic flat lesions. Subsequently, 8 asymmetric objects from plasticine were inserted in the phantom (maximum width 6-19 mm). The size of all objects (see Table 1) was measured by sliding calipers. The phantom was placed in a cylinder, $34 \mathrm{~cm}$ in diameter that was filled with water to arrive at a signal to noise ratio comparable to that in patient data. The two phantoms with lucite and plasticine polyps respectively were scanned twice: in the axial plane of the cylinder, and an orthogonal plane (see Figure 5).

Table 1. Dimensions of the phantom objects; of all lucite objects two specimens were used

\begin{tabular}{|c|c|c|}
\hline Object & Lucite Objects (lengthxheight) $[\mathrm{mm}]$ & Plasticine Objects (lengthxheight) $[\mathrm{mm}]$ \\
\hline \hline 1 & $10.0 \times 5.0$ & $19 \times 9$ \\
\hline 2 & $10.0 \times 2.5$ & $17 \times 8$ \\
\hline 3 & $8.0 \times 4.0$ & $14 \times 10$ \\
\hline 4 & $8.0 \times 2.0$ & $14 \times 8$ \\
\hline 5 & $6.0 \times 3.0$ & $12 \times 8$ \\
\hline 6 & $5.0 \times 2.5$ & $11 \times 11$ \\
\hline 7 & $4.0 \times 2.0$ & $11 \times 5$ \\
\hline 8 & - & $6 \times 5$ \\
\hline
\end{tabular}

The size of all objects was measured in the CT scans by two radiologists and by our automated method. The radiologists measured the objects in multiplanar reformatted CT images. Each object was measured twice, along the main axes as perceived by the physician. The largest value was taken as the polyp size. The automatic measurements were done as described previously. For that purpose, an arbitrary seed point was manually indicated somewhere on the polyp surface.

Inter-observer variability of the radiologists. Figure $4 \mathrm{a}, \mathrm{d}$ contains the graphs displaying the measurements of one radiologist against those of the other. 
Clearly, radiologist A tends to measure larger diameters compared to radiologist B. The average difference of their measurements was $1.2 \mathrm{~mm}$ for the lucite objects and $3.1 \mathrm{~mm}$ for the plasticine objects. The standard deviation of the absolute difference was $0.7 \mathrm{~mm}$ for the lucite objects and $2.7 \mathrm{~mm}$ for the plasticine objects.

Intra-observer variability of the radiologists and the automatic method. The intra-observer variability is assessed by the difference in size measured in the axial scan versus the measurement on the same object in the orthogonal scan. It must be conceded that this involves two different scans of the same object. We assume, however, that it allows for a good approximation of the intra-observer variability.

Figure $4 \mathrm{~b}$,e shows the measurements of the observers in one scan versus the measurement in the other. Apparently, neither the radiologists nor the automatic method shows a bias. The average absolute difference between the two measurements on the lucite objects was $1.0 \mathrm{~mm}$ and $0.8 \mathrm{~mm}$ for the two radiologists and $0.5 \mathrm{~mm}$ for the automatic technique. The standard deviation of the absolute difference was $0.9,1.0$ and $0.5 \mathrm{~mm}$ respectively. For the plasticine objects the average absolute differences were $2.6,3.2$ and $1.1 \mathrm{~mm}$ and the standard deviations $2.2,2.1$ and $1.1 \mathrm{~mm}$ respectively.

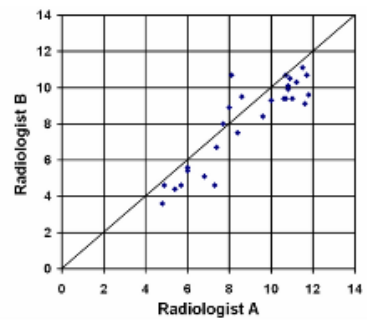

(a)

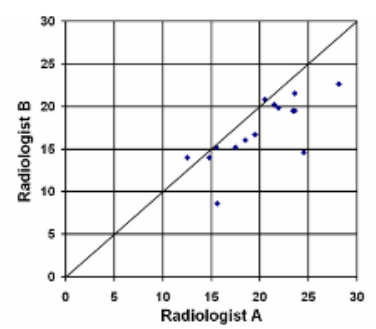

(d)

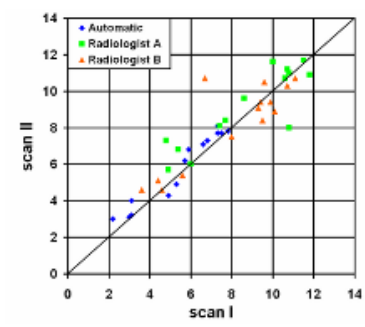

(b)

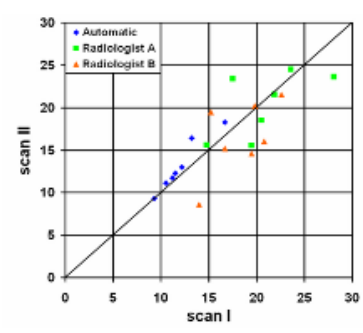

(e)

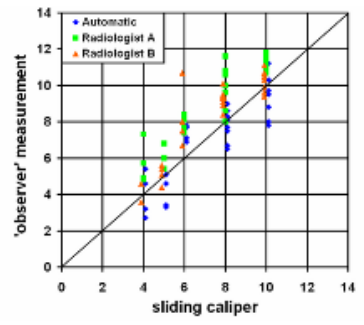

(c)

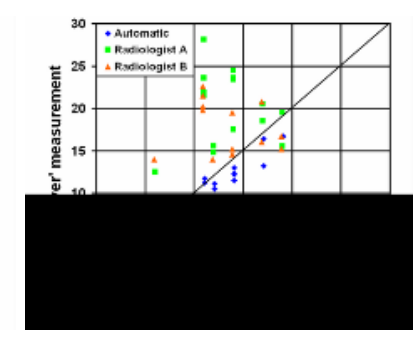

(f)

Fig. 4. First column: Inter-observer variability in polyp size for lucite (a) and plasticine (d) objects. Middle column: Intra-observer variability in polyp size for lucite (b) and plasticine (e) objects. Right column: Accuracy and precision of size measurement for lucite (c) and plasticine (f) objects. 


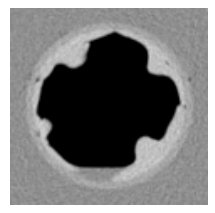

(a)

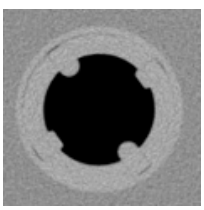

(b)

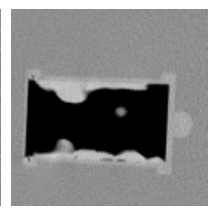

(c)

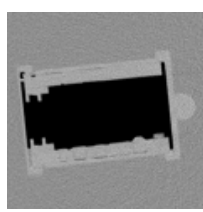

(d)

Fig. 5. Single slice from each scan. From a-d: lucite polyps axially, plasticine polyps axially, plasticine polyps orthogonally and lucite polyps orthogonally

Accuracy and precision. The accuracy and precision of the observers is defined by comparison to sliding calipers (see Figure 4k,e). The measurements of both radiologists appear to be slightly biased. This can be explained by the procedure of always selecting the larger of two measurements as the size of the polyp. The bias in the automatic method is less pronounced, but not completely absent. It can be explained by notifying that all points (except due to noise) on a segmented hemispherical polyp surface are projected inside a circle with the diameter of the polyp. An ellipse fitted through the contour points yields a small underestimation of the true size. Clearly, one might correct for all these biases in a calibration step. Specifically noticeable, is the higher precision of the automatic method on the plasticine objects, indicated by the smaller spread of values around the line of identity.

As shown in table 2, the automatic method shows a smaller systematic error than the radiologists. There is no significant difference in the precision (std. dev.) for the (symmetric) lucite objects between the automatic system and the radiologists. However, for the irregular plasticine objects the precision of the automatic system remains the same, whereas the precision of the radiologists decreases significantly.

Table 2. Mean difference and standard deviation of difference between observers and sliding calipers measurements for the lucite objects and the plasticine objects

\begin{tabular}{|c|c|c|c|c|}
\hline & \multicolumn{2}{|c|}{ Lucite } & \multicolumn{2}{c|}{ Plasticine } \\
\cline { 2 - 5 } & mean diff. [mm] & std. dev. [mm] & mean diff. [mm] & std. dev. [mm] \\
\hline Rad. A & 1.6 & 0.9 & 7 & 5 \\
\hline Rad. B & 0.8 & 1.0 & 4 & 5 \\
\hline Automatic & -0.2 & 1.2 & -1 & 1.2 \\
\hline
\end{tabular}

\section{Conclusions and Future Work}

The size of a colonographically detected polyp is important for diagnosis and decision making. The size measurement by human observers is generally considered to be imprecise and inaccurate. In this paper we presented a method for the automatic segmentation of polyp-like structures. The polyp size was automatically derived from the segmentation result. It was shown that our algorithm 
yields a smaller bias than the measurements from radiologists: on average $1 \mathrm{~mm}$ or less for the automatic method and between 1 and $7 \mathrm{~mm}$ for the radiologists, depending on the irregularity of the object. Even more important, the algorithm is consistent irrespective of the polyp shape. As opposed to that, the radiologists show a four times larger variation for the irregularly shaped objects. It is this irregularity which occurs in practice.

A good polyp segmentation algorithm is also useful for automatic polyp detection algorithms. It allows for extraction of features such as volume, surface area, average grey-value etcetera. Such features may improve the specificity of CAD algorithms.

\section{References}

1. Colorectal Cancer Facts 83 Figures Special Edition 2005. American Cancer Society, 2005.

2. Jerebko et al. Polyp segmentation method for ct colonograpy computer aided detection. Medical Imaging 2003, pages 359-169, 2003.

3. Summers RM et al. Automated polyp detection and ct colonography: feasibility assessment in a human population. Radiology, 219:51-59, 2001.

4. Yoshida H. and NSppi J. Three-dimensional computer-aided diagnosis scheme for detection of colonic polyps. IEEE Trans Med Imaging, 20(12):1261-1274, 2002.

5. Serlie I., Vos F.M., van Gelder R.E., Stoker J., Truyen R., Gerritsen F.A., Nio Y., and Post F.H. Improved visualization in virtual colonoscopy using imagebased rendering. Data Visualization 2001, Proceedings Joint Eurographics - IEEE TCVG Symposium on Visualization, pages 137-146, 2001.

6. NSppi J. and Yoshida H. Feature-guided analysis for reduction of false positives in cad of polyps for computed tomographic colonography. Med. Phys., 30(7):15921601, 2003.

7. Thoeni R.F. and Laufer I. Polyps and cancer, Textbook of Gastrointestinal Radiology. Philadelphia: W.B. Saunders, 1994.

8. Summers RM. Challenges for computer-aided diagnosis for ct colonography. $A b$ dominal Imaging, 27:268-274, 2002.

9. Hong L. Muraki S., Kaufman A., and He T. Virtual voyage: interactive navigation in the human colon. ACM SIGGRAPH Conf, pages 27-34, 1997.

10. van Wijk C, Truyen R, van Gelder RE, van Vliet LJ, and Vos FM. On normalized convolution to measure curvature features for automatic polyp detection. MICCAI 2004, part I:200-208, pages 200-208, 2004. 